\title{
Análisis de la monitorización de cinco indicadores de calidad del hemocultivo en un hospital universitario en Chile 2009-2011
}

\author{
Ana María Guzmán, Tomás Sánchez y Ricardo de la Barra
}

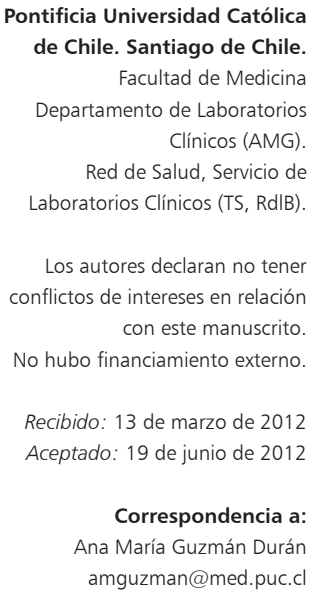

Pontificia Universidad Católica de Chile. Santiago de Chile. Facultad de Medicina Departamento de Laboratorios Clínicos (AMG).

Red de Salud, Servicio de Laboratorios Clínicos (TS, RdIB).

Los autores declaran no tener conflictos de intereses en relación con este manuscrito. No hubo financiamiento externo.

Recibido: 13 de marzo de 2012 Aceptado: 19 de junio de 2012

Correspondencia a: Ana María Guzmán Durán amguzman@med.puc.c

\author{
Quality indicators for blood culture: three years of monitoring at a \\ university hospital in Chile
}

Blood culture is considered the "gold standard" for the diagnosis of bacteremia, critical condition with high morbidity and mortality. Because of its importance, it is estimated that the blood culture is a critical test that requires close monitoring on the quality with which the process is performed. The objective of this work is to show the results of the monitoring carried out during the past three years, of 5 quality indicators of blood cultures in the laboratory of the Hospital Clínico de la Pontificia Universidad Católica de Chile, considering pre-analytical, analytical and post-analytical aspects. In the 3 years monitored the mean contamination was $0,7 \%, 46 \%$ of adult bottles had adequate volume, match between Gram stain with final identification was $99.4 \%, 100 \%$ of correct participations were achieved in surveys of external quality control and Gram staining notification before 1 hour was $88.7 \%$. With regard to proposed aims, in 2011 the laboratory complies with all, except the percentage of bottles with appropriate volume of blood inoculated. This indicator is very low and should be corrected as soon as possible since it is known that it is an important condition for optimum performance of blood cultures.

Key words: Quality indicators, blood cultures, healthcare.

Palabras clave: Indicadores de calidad, hemocultivos, seguridad asistencial.

\section{Introducción}

L as infecciones del torrente sanguíneo o bacteriemias constituyen una condición grave con alta morbi-mortalidad. Esta última varía entre 14 y $34 \%$, dependiendo de si el origen del cuadro es comunitario o asociado a la atención en salud ${ }^{1,2}$. La bacteriemia causa frecuentemente sepsis y shock séptico, por lo que la administración precoz y apropiada de antimicrobianos influye directamente en el pronóstico del paciente ${ }^{3,4}$.

El hemocultivo o cultivo microbiológico de la sangre es considerado el "estándar de oro" para el diagnóstico de bacteriemia ya que, no sólo establece la etiología infecciosa del episodio, sino que también entrega el estudio de susceptibilidad, el cual permite los ajustes de terapia necesarios ${ }^{5,6}$. Por su importancia, se estima que el hemocultivo es un examen crítico que requiere monitorización cercana respecto de la calidad con que el proceso es realizado. El Clinical and Laboratory Standards Institute (CLSI) en su guía del año 2007 y también el Cumitech publicado por la American Society for Microbiology del año 2005, sugieren algunos indicadores de calidad del hemocultivo ${ }^{7,8}$ que controlan tanto la fase pre-analítica, analítica y post-analítica.

El objetivo de este trabajo es mostrar los resultados de la monitorización de los indicadores de calidad del he- mocultivo implementados en el Laboratorio del Hospital Clínico de la Pontificia Universidad Católica de Chile, (PUC) realizada durante los últimos tres años.

\section{Materiales y Método}

Escenario: La Red de Salud UC comprende 3 centros hospitalarios, 6 centros médicos y 14 Unidades de Toma de Muestras. El Servicio de Laboratorios Clínicos de la Facultad de Medicina de la PUC, atiende la totalidad de las necesidades de exámenes de esta red, tanto de pacientes ambulatorios (385.000 al año) como hospitalizados (150.000 días/cama), realizando en total cerca de 2 millones de exámenes anuales.

Todas las botellas de hemocultivo tomadas en la Red de Salud UC entre los años 2009-2011 fueron procesadas en el Laboratorio del Hospital Clínico de la PUC utilizando el sistema automatizado BacT/ALERT® 3D (bio Mérieux SA).

Indicadores de calidad y cálculo: La Tabla 1 muestra los indicadores medidos entre los años 2009-2011 y la justificación teórica para realizar la evaluación ${ }^{7,8}$.

En la Tabla 2 se detalla la fórmula de cada indicador y la meta o umbral de cumplimiento establecido por nuestro laboratorio y/o lo aceptado internacionalmente ${ }^{8}$. 


\section{Indicador}

Fase pre-analítica

Contaminación de los hemocultivos (sangre periférica)

Botellas de hemocultivo con volumen adecuado de sangre (adultos)

Fase analítica

Concordancia de la tinción de Gram del hemocultivo con la identificación final en el cultivo.

Participaciones correctas en encuestas de control de calidad externo

Fase post-analítica
Aviso de resultados de la tinción de Gram del hemocultivo al médico tratante antes de 60 minutos (valor de alerta)

\section{Justificación}

Hemocultivos contaminados traducen mala técnica de antisepsia de la piel, provocan gastos innecesarios de tiempo y recursos, en el procesamiento de botellas contaminadas e inducen retraso en el diagnóstico de infecciones del torrente sanguíneo.

Se considera un hemocultivo contaminado, si uno de dos frascos de cada set de hemocultivo presenta desarrollo de microorganismos tales como: Staphylococcus coagulasa negativa, Propionibacterium sp., Bacillus sp., Corynebacterium sp., Streptococcus grupo viridans, Clostridium sp., y Micrococcus sp.

El volumen de sangre obtenido del paciente para cultivo es la variable más importante para lograr el máximo rendimiento del hemocultivo. Se considera que el volumen óptimo de sangre a colocar en las botellas de adultos es de $10 \mathrm{~mL}$.

Una correcta lectura de la tinción de Gram es crítica para el inicio y ajuste de terapia antimicrobiana en pacientes con bacteriemia.

Resultados correctos en las Encuestas de Control de Calidad Externo (ensayos de aptitud) contribuyen a asegurar la calidad de los resultados generados en el laboratorio.

La presencia de crecimiento bacteriano a partir de una botella de hemocultivo debe ser considerada como valor de alerta para el médico, ya que permite orientar la terapia. Se avisa la positividad del hemocultivo en conjunto con la morfología, agrupación y afinidad tintorial de las bacterias visualizadas en la tinción de Gram.

\section{Tabla 2. Fórmula y meta o umbral de cumplimiento de los indicadores del hemocultivo implementados en el Laboratorio del Hospital Clínico PUC}

\section{Indicador}

$\%$ de contaminación de los hemocultivos de sangre

periférica (SP)

$\%$ de botellas de hemocultivo con volumen adecuado (adultos)

\% de concordancia de la tinción de Gram del hemocultivo con la identificación final

$\%$ de participaciones correctas en encuestas de control de calidad externo (Blood Culture Survey, CAP)

$\%$ de aviso de resultados de la tinción de Gram del hemocultivo al médico tratante antes de 60 minutos como valor de alerta

\section{Fórmula}

$\mathrm{n}^{\circ}$ botellas hemocultivo contaminadas (SP) $\times 100$
$\mathrm{n}^{\circ}$ total de botellas de hemocultivo (SP) tomadas

$n^{\circ}$ botellas hemocultivo de adulto con volumen adecuado $\mathrm{n}^{\circ}$ total botellas hemocultivo de adulto tomadas

$\mathrm{n}^{\circ}$ láminas Gram hemocultivo concordantes con cultivo $\times 100$

$n^{\circ}$ total láminas de Gram de hemocultivos realizados $n^{\circ}$ determinaciones aceptadas en encuesta $\times 100$

$\mathrm{n}^{\circ}$ total determinaciones realizadas en encuesta

$n^{\circ}$ de tinciones de Gram del hemocultivos positivos avisadas antes de los $60 \mathrm{~min}$

$\mathrm{n}^{\circ}$ total de tinciones de Gram del hemocultivos positivos realizadas
Meta

$\leq 2 \%$

$\geq 80 \%$

$\geq 98 \%$

$\geq 97 \%$

(\%: porcentaje, no: número, SP: sangre periférica, CAP: Colegio de Patólogos Americanos)

A continuación se detalla el método de obtención de los datos para cada uno de ellos:

- Porcentaje de contaminación: Según la literatura médica disponible y ampliamente consensuada ${ }^{7}$ se consideró contaminación si uno de dos o más frascos del hemocultivo tomado por cada paciente presentaba desarrollo de alguno de los siguientes microorganismos: Staphylococcus coagulasa negativa, Propionibacterium spp., Bacillus spp., Corynebacterium spp., Streptococcus grupo viridans, Clostridium spp., y Micrococcus spp.

- Porcentaje de botellas con volumen adecuado de sangre inoculado: El volumen inoculado en cada botella se calculó pesando cada botella al ingreso al laboratorio y extrapolando el peso en una tabla peso vs volumen entregada y validada por el fabricante. El volumen de sangre en cada botella se clasificó como óptimo si contenía 5-10 mL de sangre, como escaso con menos de $5 \mathrm{~mL}$ y excesivo con más de $10 \mathrm{~mL}$.

- Concordancia del resultado de la tinción de Gram con la identificación final en el cultivo: Se consideró discordancia absoluta el informe equivocado, ya fuese de morfología o afinidad tintorial en el Gram, respecto al cultivo y discordancia parcial el crecimiento de un segundo microorganismo no visualizado en la tinción de Gram.

- Porcentaje de participaciones correctas en encuestas de control de calidad externo (ensayos de aptitud): Debido a que en el país no existen encuestas dirigidas específicamente al hemocultivo, nuestro laboratorio 


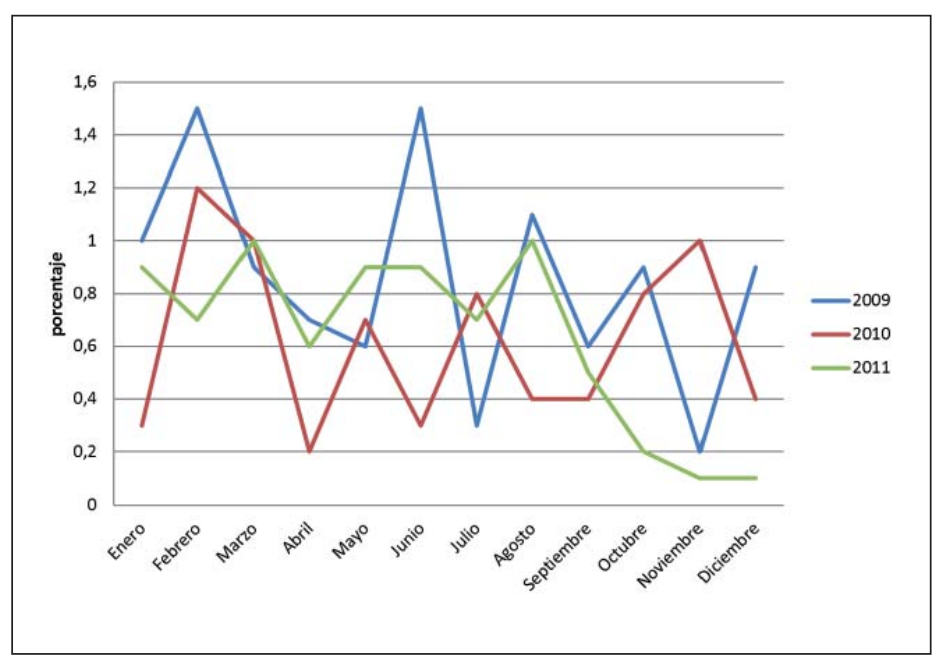

Figura 1. Porcentaje de contaminación mensual del hemocultivo (2009-2011).

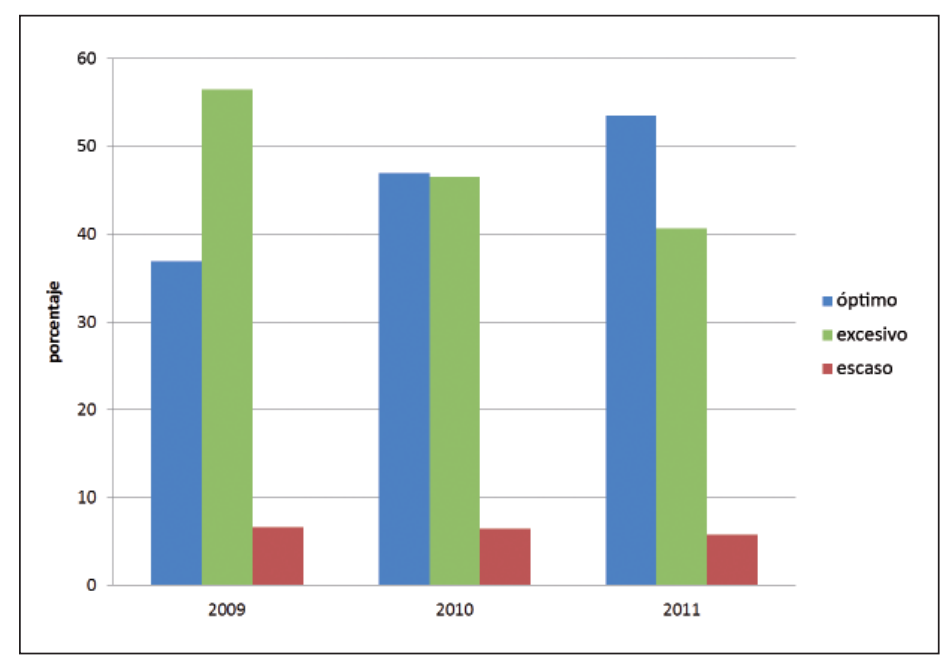

Figura 2. Distribución porcentual de botellas de hemocultivo de sangre periférica con volumen adecuado, excesivo y escaso (2009-2011). participa en una encuesta internacional que corresponde al "Blood Culture Survey" del College of American Pathologists (CAP) que evalúa, en 8 muestras anuales, resultados de crecimiento, tinción de Gram e identificación final de microorganismo.

\begin{tabular}{|c|c|c|c|}
\hline & Informe final & Informe de Tinción Gram & Tipo de discordancia \\
\hline 1 & $\begin{array}{l}\text { Staphylococcus coagulasa negativa } \\
\text { Pseudomonas aeruginosa }\end{array}$ & $\begin{array}{l}\text { Cocáceas Gram positivas en } \\
\text { racimo }\end{array}$ & Parcial \\
\hline 2 & $\begin{array}{l}\text { Staphylococcus aureus } \\
\text { Candida albicans }\end{array}$ & $\begin{array}{l}\text { Cocáceas Gram positivas en } \\
\text { racimo }\end{array}$ & Parcial \\
\hline 3 & $\begin{array}{l}\text { Staphylococcus coagulasa negativa } \\
\text { Salmonella Enteritidis }\end{array}$ & $\begin{array}{l}\text { Cocáceas Gram positivas en } \\
\text { racimo }\end{array}$ & Parcial \\
\hline 4 & $\begin{array}{l}\text { Staphylococcus aureus } \\
\text { Escherichia coli }\end{array}$ & $\begin{array}{l}\text { Cocáceas Gram positivas en } \\
\text { racimo }\end{array}$ & Parcial \\
\hline 5 & $\begin{array}{l}\text { Staphylococcus coagulasa negativa } \\
\text { Acinetobacter baumannii }\end{array}$ & $\begin{array}{l}\text { Cocáceas Gram positivas en } \\
\text { racimo }\end{array}$ & Parcial \\
\hline 6 & $\begin{array}{l}\text { Streptococcus grupo viridans } \\
\text { Escherichia coli }\end{array}$ & $\begin{array}{l}\text { Cocáceas Gram positivas } \\
\text { aisladas y en cadenas }\end{array}$ & Parcial \\
\hline 7 & $\begin{array}{l}\text { Enterococcus faecium } \\
\text { Acinetobacter baumannii }\end{array}$ & $\begin{array}{l}\text { Cocáceas Gram positivas en } \\
\text { pares y cadenas }\end{array}$ & Parcial \\
\hline 8 & $\begin{array}{l}\text { Enterococcus faecalis } \\
\text { Candida albicans }\end{array}$ & $\begin{array}{l}\text { Cocáceas Gram positivas en } \\
\text { pares y cadenas }\end{array}$ & Parcial \\
\hline 9 & $\begin{array}{l}\text { Enterococcus faecalis } \\
\text { Candida albicans }\end{array}$ & $\begin{array}{l}\text { Cocáceas Gram positivas en } \\
\text { pares y cadenas }\end{array}$ & Parcial \\
\hline 10 & $\begin{array}{l}\text { Enterococcus faecalis } \\
\text { Candida albicans }\end{array}$ & $\begin{array}{l}\text { Cocáceas Gram positivas en } \\
\text { pares y cadenas }\end{array}$ & Parcial \\
\hline 11 & $\begin{array}{l}\text { Enterococcus faecalis } \\
\text { Candida albicans }\end{array}$ & $\begin{array}{l}\text { Cocáceas Gram positivas en } \\
\text { pares y cadenas }\end{array}$ & Parcial \\
\hline 12 & $\begin{array}{l}\text { Candida albicans } \\
\text { Escherichia coli }\end{array}$ & Levaduras & Parcial \\
\hline 13 & $\begin{array}{l}\text { Candida albicans } \\
\text { Escherichia coli }\end{array}$ & Levaduras & Parcial \\
\hline 14 & Corynebacterium spp. & Cocáceas Gram positivas & Absoluta \\
\hline
\end{tabular}

- Porcentaje de aviso de resultados de la tinción de Gram del hemocultivo al médico tratante antes de 60 minutos como valor de alerta: el Laboratorio lleva un registro y base de datos (construida en base Access, Microsoft ${ }^{\circledR}$ ) de donde se extrajo el tiempo transcurrido en el aviso de los valores de alerta al médico tratante.

\section{Resultados}

Porcentaje de contaminación: en la Figura 1 se muestran los porcentajes de contaminación obtenidos de la medición mensual 2009-2011. En estos tres años se procesaron 25.000 frascos de hemocultivos, de los cuales 176 resultaron contaminados, por lo que la contaminación global fue $0,7 \%$, cifra que se encuentra muy por debajo de la meta fijada $\leq 2 \%$,

Porcentaje de botellas de hemocultivo con volumen apropiado de sangre inoculada: el porcentaje de botellas con volumen apropiado de sangre se mantuvo muy bajo durante los tres años analizados, logrando una cifra global de 46,1\%. Prácticamente la mitad de los frascos (47,6\%) se inocularon con volúmenes excesivos (Figura 2).

Concordancia del resultado de la tinción de Gram con la identificación final en el cultivo: Durante los tres años se detectó un alto nivel de concordancia, superior a $99 \%$, con sólo 14 láminas discordantes de un total de 2.258 procesadas. De estas láminas, 13 correspondieron a discordancias parciales, en las cuales los hemocultivos positivos a más de un microorganismo fueron informados con sólo una morfología microbiana en la tinción de Gram. En sólo un caso hubo discordancia absoluta con el microorganismo identificado: un Corynebacterium sp. que fue informado como cocácea Gram positiva (Tabla 3). 
Porcentaje de participaciones correctas en encuestas de control de calidad externo (ensayos de aptitud): Durante los tres años se realizó el análisis de 24 muestras, cuyos resultados fueron $100 \%$ concordantes para crecimiento, tinción de Gram y cultivo.

Porcentaje de aviso del resultado de la tinción de Gram del hemocultivo al médico tratante antes de 60 minutos como valor de alerta: Durante el año 2009 y 2010 se avisaron 86 y $88,8 \%$ de las tinciones de Gram antes de 60 minutos, respectivamente. El año 2011 se logró superar la meta (90\%) avisando en el plazo a 91\% de los casos (Tabla 4).

\section{Discusión}

Los laboratorios clínicos microbiológicos tienen un importante rol detectando los microorganismos patógenos en el hemocultivo e informando correctamente el estudio de susceptibilidad, para orientar al médico en el manejo de la bacteriemia y selección de antimicrobianos.

La implementación de indicadores de calidad para la monitorización del desempeño de este examen se considera necesaria. Es así como por varios años hemos estado familiarizados con la monitorización del porcentaje de contaminación de los hemocultivos, característica evaluada en el programa de acreditación del Ministerio de Salud en el contexto del manejo de las infecciones asociadas a la atención en salud en nuestro país. Posteriormente, en la literatura médica se ha sugerido la implementación de otros indicadores con el fin de no sólo monitorizar la fase pre-analítica, sino también, la fase analítica y post-analítica del hemocultivo, como son los estudiados en este trabajo.

Contaminación de los hemocultivos. Este indicador corresponde a un parámetro crítico para obtener resultados confiables, ya que hemocultivos tomados con mala técnica de antisepsia y que resultan contaminados requerirán repeticiones del examen provocando retraso en el diagnóstico del paciente y en ocasiones uso innecesario o inadecuado de antimicrobianos. Internacionalmente se recomienda que el porcentaje de contaminación del hemocultivo debe ser menor a $2 \%^{7-10}$. En dos estudios multicéntricos que incluyeron 600 y 300 hospitales, la tasa de contaminación varió de 2,5 a 2,9\%, respectivamente ${ }^{9,10}$. Nuestro porcentaje de contaminación se encuentra siempre bajo $1 \%$ indicando una buena técnica de toma de muestra.

Hemocultivos con volumen adecuado de sangre. El cumplimiento de este indicador, aunque presentó en los tres años evaluados una mejoría discreta desde 36,9 a $53,5 \%$, de todas formas está muy lejos de la meta plantea-

\begin{tabular}{|ccc|}
\hline \multicolumn{2}{|c|}{ Tabla 4. Porcentaje de aviso de resultados de la tinción de Gram del hemocultivo } \\
al médico tratante antes de 60 minutos \\
Año & $\begin{array}{c}\text { Número de valores de alerta } \\
\text { provenientes del hemocultivo }\end{array}$ & $\begin{array}{c}\text { Porcentaje de aviso antes de los } 60 \\
\text { minutos }\end{array}$ \\
\hline 2009 & 932 & $86,0 \%$ \\
2010 & 887 & $88,8 \%$ \\
2011 & 971 & $91,0 \%$ \\
\hline Total & 2.790 & $88,7 \%$ \\
\hline
\end{tabular}

da de tener al menos $80 \%$ de las botellas inoculadas apropiadamente. El principal problema para el cumplimiento de este indicador es que las botellas de hemocultivo tienen un vacío mayor que el correspondiente a $10 \mathrm{~mL}$. Lamentablemente, la factibilidad de producir botellas con vacíos estandarizados para los volúmenes de sangre requeridos para el hemocultivo es hasta ahora técnicamente imposible para la industria de estos insumos. Se sabe que si se altera la relación sangre:medio de cultivo, tanto por volúmenes escasos como excesivos, el rendimiento del hemocultivo no será óptimo ${ }^{11}$. Esta situación debe ser afrontada conjuntamente con el estamento de enfermería en todos los Servicios Clínicos a nivel para mejorar lo más pronto posible este indicador. En este trabajo, los datos de los hemocultivos tomados en los servicios de pacientes pediátricos no fueron analizados, ya que son difíciles de interpretar, especialmente en unidades de pacientes prematuros o de muy bajo peso al nacimiento, en los cuales la volemia es muy menor y colocar metas de cumplimiento es muy difícil.

Concordancia del Gram del hemocultivo con la identificación final en el cultivo. La tinción de Gram se considera el test más importante en el manejo de un hemocultivo positivo, por su rapidez en la caracterización del microorganismo y de esta forma permite orientar al médico en el uso de antimicrobianos precozmente ${ }^{12}$. Este indicador está dirigido a evitar el inicio de tratamientos empíricos basados en una tinción de Gram informada erróneamente. La competencia del tecnólogo médico que observa la preparación es fundamental en el cumplimiento de este indicador. En centros que han vigilado el problema por más de 10 años, se ha informado una discordancia que oscila entre 1 y $5 \%{ }^{13-15}$, cifra que en nuestro laboratorio es menor al 1\%. Si consideramos sólo la discordancia absoluta (Corynebacterium sp. informado como cocácea Gram (+)) la discordancia fue $0,04 \%$ en tres años.

Participaciones correctas en encuestas de control de calidad externo. Las encuestas de control de calidad externo o ensayos de aptitud es una importante herramienta para asegurar resultados seguros y confiables en todas las áreas del laboratorio clínico, incluida la Microbiología. En 
estos tres años, todos los resultados de la encuesta para la evaluación de hemocultivos (Blood Culture Survey) generados por nuestro laboratorio fueron clasificados como satisfactorios. Según lo publicado en la literatura médica, la concordancia esperada en microbiología bordea el $93 \%{ }^{16}$.

Aviso del resultado de la tinción de Gram del hemocultivo al médico tratante antes de los 60 minutos como valor de alerta. Los valores de alerta corresponden a resultados que involucran riesgo para la vida del paciente por lo que deben ser avisados lo antes posible al médico tratante ${ }^{17}$. En nuestro hospital, siguiendo las recomendaciones del Clinical and Laboratory Standards Institute, el tiempo fijado para avisar la tinción de Gram de un hemocultivo identificado como positivo es de 1 hora. Trabajos recientes han demostrado que el aviso dentro de este lapso está asociado a una disminución de la mortalidad de los pacientes ${ }^{18}$. En el año 2011, a diferencia de los años anteriores, nuestro laboratorio logró estar por sobre la meta, con la implementación de un cronómetro para el tecnólogo médico, que comienza a medir el tiempo desde que el equipo de hemocultivos avisa la positividad del frasco. Sin embargo, creemos que los tiempos de aviso pueden acortarse aún más con la incorporación de sistemas informáticos de laboratorio más modernos que consideren la mensajería a los médicos tratantes en forma automática.

\section{Comentario final}

La incorporación de indicadores de calidad para la monitorización de procesos críticos dentro del laboratorio, constituye indudablemente una oportunidad de identificar áreas de mejora. Localmente debemos preocuparnos por mejorar la toma de muestra de los hemocultivos respecto al volumen de sangre inoculado y también lograr menores tiempos de aviso para el valor de alerta de la tinción de Gram de los hemocultivos.

Un área no evaluada a través de los indicadores aquí presentados es la competencia del médico tratante, tanto en la solicitud como interpretación de los resultados del hemocultivo. Sería interesante obtener, por ejemplo, datos de proporción de pacientes ingresados con sospecha de neumonía con solicitud de hemocultivo, lo cual será posible en la medida que las instituciones adopten o desarrollen guías clínicas basadas en el conocimiento científico, sólidas y consensuadas ojalá internacionalmente, para el diagnóstico y manejo de diversas patologías.

\section{Resumen}

El hemocultivo es el "estándar de oro" para el diagnóstico de bacteriemia, condición grave de alta morbimortalidad. Por esto, se estima que el hemocultivo es un examen crítico, que requiere monitorización de su calidad. El objetivo de este trabajo es mostrar los resultados de la monitorización de 3 años de 5 indicadores de calidad del hemocultivo implementados en nuestro hospital. El porcentaje promedio de contaminación de las botellas para hemocultivo fue $0,7 \%$, el porcentaje de botellas de adultos con volumen adecuado fue $46 \%$, la concordancia de la tinción de Gram con la identificación final correspondió a 99,4\%, el 100\% de las participaciones en encuestas de control de calidad externo fueron correctas y $88,7 \%$ de los avisos de valores de alerta de la tinción de Gram fueron realizados antes de 1 hora. En el año 2011 se cumplió con las metas propuestas por el laboratorio para todos los indicadores, excepto con el porcentaje de botellas con volumen apropiado de sangre. Este último, se encuentra muy por debajo de la meta y debe ser mejorado a la brevedad, ya que el volumen de sangre cultivada es el factor más importante para obtener un rendimiento óptimo del hemocultivo.

\section{Referencias bibliográficas}

1.- Riedel S, Carroll K. Blood cultures: key elements for best practices and future directions. J Infect Chemother 2010; 16: 301-16.

2.- Diekema D J, Beekmann S E, Chapin K C, Morel K A, Munson E, Doern G V. Epidemiology and outcome of nosocomial and community-onset bloodstream infection J Clin Microbiol 2003; 41: 3655-60.

3.- Bearman G, Wenzel R. Bacteremias: a leading cause of death. Arch Med Res 2005; 36: 646-59.

4.- Wisplinghoff $\mathrm{H}$, Bischoff T, Tallent S, Seifert H, Wenzel R, Edmond M. Nosocomial bloodstream infections in US hospitals: analysis of 24,179 cases from a prospective nationwide surveillance study. Clin Infect Dis 2004; 39 :
309-17.

5.- Magadia R, Weinstein M. Laboratory diagnosis of bacteremia and fungemia. Infect Dis Clin North Am 2001; 15: 1009-24.

6.- Pien B, Sundaram P, Raoof N, Costa S, Mirrett S, Woods C, et al. The clinical and prognostic importance of positive blood cultures in adults. Am J Med 2010; 123: 819-28.

7.- Clinical and Laboratory Standards Institute (CLSI). Principles and Procedures for Blood Cultures; Approved guideline. CLSI Document M47-A (2007).

8.- Baron E, Weinstein M, Dunne M, Yagupski P, Welch D, Wilson D. Cumulative Techniques and Procedures in Clinical Microbiology (Cumitech) 1C: Blood Cultures IV. ASM Press (2005)

9.- Schifman R, Strand C, Meier F, Howanits P.
Blood culture contamination: a College of American Pathologists Q-Probes study involving 640 institutions and 497134 specimens from adult patients. Arch Pathol Lab Med 1998; 122: 216-21.

10.- Bekeris L, Tworek J, Walsh M, Valenstein P. Trends in blood culture contamination: a College of American Pathologists Q-Tracks study of 356 institutions. Arch Pathol Lab Med. 2005; 129: 1222-5.

11.- Weinstein M P, Mirrett S, Wilson M L, Reimer L G, Reller L B. Controlled evaluation of 5 versus 10 milliliters of blood cultured in aerobic BacT/Alert blood culture bottles. J Clin Microbiol 1994; 32: 2103-6.

12.- Uehara Y, Yagoshi M, Tanimichi Y, Yamada H, Shimoguchi K, Yamamoto S, et al. Impact of 
reporting gram stain results from blood culture bottles on the selection of antimicrobial agents. Am J Clin Pathol 2009; 132: 18-25.

13.- Rand K, Tillan M. Errors in interpretation of Gram stains from positive blood cultures. Am J Clin Pathol 2006; 126: 686-90.

14.- Sogaard M, Norgaard M, Schonheyder H C. First notification of positive blood cultures and high accuracy of the Gram stain report. J Clin Microbiol 2007; 45: 1113-17.

15.- Strand C. Positive blood cultures. Can we always trust the Gram stain? Am J Clin Pathol 2006; 126: 671-2.

16.- Shahangian S, Snyder S. Laboratory medicine quality indicators. A review of the literature. Am J Clin Pathol 2009; 131: 418-31.
17.- Lundberg G. When to panic over abnormal values. MLO Med Lab Observ 1972; 4: 47-54.

18.- Barenfanger J, Graham D, Kolluri L, Sangwan G, Lawhorn J, Drake C, et al. Decreased mortality associated with prompt Gram staining of blood cultures. Am J Clin Pathol 2008; 130: 870-6. 